\title{
Unified Approach for Robust Stability Analysis of Buck Converters with Discrete-Time Sliding Mode Control
}

\author{
Yanmin Wang $\mathbb{D}^{1},{ }^{1}$ Yang Ge $\mathbb{D D}^{1},{ }^{1}$ Ping Zhang $\mathbb{D}^{2},{ }^{2}$ and Guangxin Duan $\mathbb{D}^{1}$ \\ ${ }^{1}$ School of Electrical Engineering and Automation, Harbin Institute of Technology, Harbin 150001, China \\ ${ }^{2} 716$ Research Institute, China Shipbuilding Industry Corporation, Lianyungang 222006, China
}

Correspondence should be addressed to Yanmin Wang; wangyanmin@hit.edu.cn

Received 1 February 2021; Accepted 9 June 2021; Published 19 June 2021

Academic Editor: Yuqiang Wu

Copyright ( 2021 Yanmin Wang et al. This is an open access article distributed under the Creative Commons Attribution License, which permits unrestricted use, distribution, and reproduction in any medium, provided the original work is properly cited.

This paper proposes a novel discrete-time sliding mode (DTSM) control approach to address the robust stability problem of buck converters with multiple disturbances. The contributions lie in the "unified" modelling and controller design. In modelling, all the possible model uncertainties and external disturbances are considered and further classified into two cases. It can also be extended to the situations with individual/several disturbances. While for the controller design, differing from the traditional DTSM based on the nominal model, the disturbances are directly introduced in the process, giving the robust stability condition and four new regulation subranges. It is suitable for both nominal and perturbed systems. Finally, the influences of the sampling time and disturbances on the control performance are investigated. Simulations and experiments confirm the benefits of the unified approach with greater accuracy and wider applications.

\section{Introduction}

The issue of model uncertainty and external disturbance is widespread and challenging for the control of power converters. It might be related to the internal circuit elements, input power supply, measurement device, external environment interference, and so on [1-3]. For example, in [4], the magnetic characteristics of inductor are proved to vary greatly in presence of large magnetic flux density; in [5], the variations of load resistor and input voltage are proved not to be neglected, especially in case of large signal operation.

As a kind of favourite DC/DC power converters, buck converters have been widely used in renewable energy systems, distributed generation systems, marine power plants, and so on $[6,7]$. They are characterized as nonlinear and time-varying systems with model uncertainties and external disturbances. In modelling, many efforts have been centred on diverse kinds of system descriptions and auxiliary observers [8-12]. In [1], the commonly used average statespace model is given. In [13], the disturbances of load resistor, inductor, and capacitor are considered and a unified linear fractional model with structured dynamic uncertainties is proposed. In [11], a stochastic system model is proposed for buck converters with noise and load disturbance. In [12], a Hammerstein model is proposed for the case of duty-cycle disturbance. However, at present, many researchers only focus on one or several disturbing factors in practical applications. Although as an alternative solution auxiliary observers are generally adopted to relieve the burden of high-precision modelling $[9,14,15]$, it increases the system complexity and can still not cover all the possible model uncertainties and external disturbances.

For the control of buck converters, the pulse width modulation (PWM) is a conventional approach but suffers from the bottleneck of model precision, especially when facing the possible disturbances $[1,9,13,16]$. As an effective robust control substitute, sliding mode (SM) control technology has been widely adopted with advantages of implementation simplicity, guaranteed stability, and fast response [1,2, 17-20]. A survey of recent SM applications in power converters can be referred to $[1,20]$. The current research mainly focuses on designing the resistant-disturbance controllers and compensators. For the former, it is often used to overcome some kinds of matching 
disturbances directly $[2,17,19]$; while for the latter, it is generally used for some specific mismatching disturbances [21-23], which can not satisfy the matching condition of SM $[1,2,21-23]$. However, due to the diversity and complexity of model uncertainties and external disturbances of converters, whether there is a unified approach for the design and analysis of SM controllers is an interesting problem worthy of consideration but has been barely investigated.

With the rapid development of programmed microprocessor chips, the discretization is another issue not to be avoided for SM controlled converters [24-26]. In general, the discretization realization includes two steps: designing an appropriate control algorithm in continuous-time domain $[1,17]$ and further making an analogue-to-digital transformation for the corresponding digital controller [27-29]. In order to guarantee the stability of discrete-time sliding mode (DTSM) control systems, the sampling time and controller parameter are proved to be the two key factors [24, 29-32]. For the former, the smaller the sampling time is, more closely the DTSM system approximates to the original continuous-time counterpart. However, it increases the hardware costs and computational burdens. For the latter, due to the inherent switching nonlinearity of SM control, how to regulate the controller parameters is still an unsettled problem. In [24, 28-30], it has proved that inappropriate discretization can aggravate the control performance, leading to low accuracy and high wear of physical parts, even to the point of causing instability.

Based on the above analysis, a unified approach is proposed to address the robust stability problem of DTSM controlled buck converters with multiple disturbances. How to regulate the controller parameter and sampling time? What are the disturbances that affect the quality of the output voltage? They are two key questions. As a result, a unified approach of modelling and design of DTSM controller is proposed. To be specific, the contributions of this paper can be concluded as follows: (1) all possible model uncertainties and external disturbances of buck converters are considered in modelling and control, including the variations of input and reference output voltage, parameter uncertainties of load resistor, inductor, and capacitor, and time-varying external disturbances; (2) differing from the traditional DTSM approach $[1,24,27,31]$, four more accurate subranges of controller parameter can be achieved by the stability analysis; and (3) the influences of the sampling time and disturbances on the controller regulation and control performance are investigated simultaneously.

The structure of this paper is organized as follows. In Section 2, the system description of SM controlled buck converter under multiple disturbances is given, following the unified disturbance analysis and discretization of the system. In Section 3, a unified approach of DTSM controller is proposed by the robust stability analysis, following the influences of the sampling time and disturbances on the system. The simulations and experiments are presented in Section 4 and concluded in Section 5 to validate the proposed approach with greater accuracy and wider applications.
Notations. The following notations are used throughout this paper. $T$ denotes the transpose of the matrix, $I \in R^{n}$ denotes the $n \times n$ identity matrix, $O($.) denotes the infinitesimal term of the corresponding parameter, and the items with " $\Delta$ " represent the disturbances of the corresponding nominal variables.

\section{System Description}

Figure 1 gives the system diagram of a SM controlled DC/ DC buck converter $[1,17,19]$. In the converter circuit, $E$ is the input DC voltage source; $L, C$, and $R$ are the inductor, capacitor, and load resistor; $i_{L}$ and $i_{C}$ are the currents flowing through $L$ and $C$, respectively; $v_{c}$ is the output voltage; $D$ is a diode; $S_{w}$ is a power switch; $V_{\text {ref }}$ is the DC reference voltage of $v_{c}$; the states $x_{1}$ and $x_{2}$ are defined as the output voltage error and its derivative as follows:

$$
\left\{\begin{array}{l}
x_{1}=v_{c}-V_{\text {ref }}, \\
x_{2}=\dot{x}_{1}=\dot{v}_{c}-\dot{V}_{\text {ref }}=\dot{v}_{c} .
\end{array}\right.
$$

To realize the ON/OFF control of the power switch $S_{w}$, this paper directly adopts the inherent switching characteristics of SM control as follows $[1,2]$ :

$$
u=-\frac{1}{2}[\operatorname{sgn}(s)-1],
$$

which is implemented by the hysteresis circuit $[1,19] ; u=1$ and $u=0$ are used to denote the status of switch ON and $\mathrm{OFF}$, respectively; $s$ is the SM variable designed later.

2.1. Modelling the Buck Converter under Disturbances. Here, we assume that the buck converter works in continuous conduction mode. Based on the average state-space modelling approach and Kirchhoff's circuit law, the ON/ OFF operations of the buck converter in Figure 1 can be described, respectively, as

$$
\begin{aligned}
& \left\{\begin{array}{l}
\frac{\mathrm{d} i_{L}}{\mathrm{~d} t}=\frac{1}{L}\left(E-v_{c}\right), \\
\frac{\mathrm{d} v_{c}}{\mathrm{~d} t}=\frac{1}{C}\left(i_{L}-\frac{v_{c}}{R}\right),
\end{array}\right. \\
& \left\{\begin{array}{l}
\frac{\mathrm{d} i_{L}}{\mathrm{~d} t}=-\frac{1}{L} v_{c}, \\
\frac{\mathrm{d} v_{c}}{\mathrm{~d} t}=\frac{1}{C}\left(i_{L}-\frac{v_{c}}{R}\right) .
\end{array}\right.
\end{aligned}
$$

By combining (3) and (4), it yields

$$
\left\{\begin{array}{l}
\frac{\mathrm{d} i_{L}}{\mathrm{~d} t}=\frac{1}{L}\left(u E-v_{c}\right), \\
\frac{\mathrm{d} v_{c}}{\mathrm{~d} t}=\frac{1}{C}\left(i_{L}-\frac{v_{c}}{R}\right) .
\end{array}\right.
$$




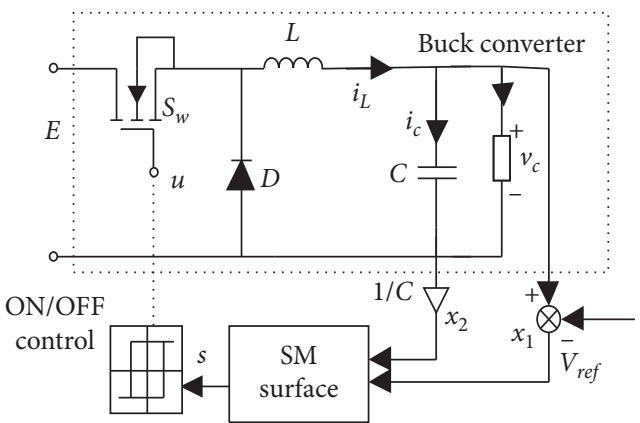

Figure 1: Diagram of a SM controlled buck converter system.

$$
\left\{\begin{array}{l}
\frac{\mathrm{d} i_{L}}{\mathrm{~d} t}=\frac{1}{L+\Delta L}\left[u(\Delta E+E)-v_{c}\right]+d_{1}(t) \\
\frac{\mathrm{d} v_{c}}{\mathrm{~d} t}=\frac{1}{C+\Delta C}\left(i_{L}-\frac{v_{c}}{R+\Delta R}\right)+d_{2}(t)
\end{array}\right.
$$

where the items with " $\Delta$ " are the corresponding disturbances of the nominal variables $R, E, L$, and $C$ and $d_{1}(t)$ and $d_{2}(t)$ are the external disturbances, and their time derivatives are assumed bounded.

Here, we define $\Delta V_{\text {ref }}$ as the variation of the reference output voltage $V_{\text {ref. }}$ By combining (1), (6) can be further changed as

In this paper, we consider all the possible model uncertainties and external disturbances. Therefore, (5) can be changed as

$$
\left\{\begin{array}{l}
\dot{x}_{1}=x_{2}, \\
\dot{x}_{2}=-\frac{x_{2}}{R C}+\frac{1}{L C}\left(u E-V_{\text {ref }}-x_{1}\right)-\frac{1}{L C} \Delta V_{\text {ref }}+\frac{\omega_{1}(t)}{C}-\frac{\omega_{2}(t)}{R C},
\end{array}\right.
$$

where the terms $\omega_{1}(t)$ and $\omega_{2}(t)$ are denoted as

$$
\left\{\begin{array}{l}
\omega_{1}(t)=\frac{\Delta E L C u+(\Delta L C+\Delta C L+\Delta L \Delta C)\left(v_{C}-u E+\Delta V_{\mathrm{ref}}\right)}{L(L+\Delta L)(C+\Delta C)}+\frac{C}{C+\Delta C} d_{1}(t), \\
\omega_{2}(t)=\frac{R \Delta C+\Delta R C+\Delta R \Delta C}{(R+\Delta R)(C+\Delta C)}\left(\frac{i_{L}}{C}-\frac{v_{c}}{R C}\right)+R C \dot{d}_{2}(t) .
\end{array}\right.
$$

Rewrite (8) in the form of state space as follows:

$$
\dot{x}=\mathbf{A x}+[\mathbf{B}+\Delta \mathbf{B}] u+\left[\mathbf{D}+\Delta \mathbf{D}_{1}+\Delta \mathbf{D}_{2}(t)+\Delta \mathbf{D}_{3}(\mathbf{x})\right],
$$

where the state $x=\left[x_{1}, x_{2}\right]^{T}$ and the nominal matrix $A, B$, and $D$ are listed as

$$
\begin{aligned}
& \mathbf{A}=\left[\begin{array}{cc}
0 & 1 \\
-\omega_{0}^{2} & -\frac{1}{R C}
\end{array}\right], \\
& \mathbf{B}=\left[\begin{array}{c}
0 \\
\omega_{0}^{2} E
\end{array}\right], \\
& \mathbf{D}=\left[\begin{array}{c}
0 \\
-V_{\mathrm{ref}} \omega_{0}^{2}
\end{array}\right],
\end{aligned}
$$

and $\Delta B, \Delta D_{1}, \Delta D_{2}(t)$, and $\Delta D_{3}(x)$ represent the lumped input disturbance, constant disturbance, time-varying disturbance, and state disturbance deduced as

$$
\Delta \mathbf{B}=\left[\begin{array}{c}
0 \\
\frac{\Delta E L C-\Delta L E C-\Delta C L E-\Delta L \Delta C E}{L C(L+\Delta L)(C+\Delta C)}
\end{array}\right]
$$

$$
\begin{aligned}
\Delta \mathbf{D}_{1} & =\left[\begin{array}{c}
0 \\
\frac{\Delta L C V_{\mathrm{ref}}+\Delta C L V_{\mathrm{ref}}+\Delta L \Delta C L V_{\mathrm{ref}}-L C \Delta V_{\mathrm{ref}}}{L C(L+\Delta L)(C+\Delta C)}
\end{array}\right], \\
\Delta \mathbf{D}_{2}(t) & =\left[\begin{array}{c}
0 \\
\frac{d_{1}(t)}{C+\Delta C}+\dot{d}_{2}(t)
\end{array}\right], \\
\Delta \mathbf{D}_{3}(\mathbf{x}) & =\left[\begin{array}{c}
\frac{R \Delta C+\Delta R C+\Delta R \Delta C}{R C(R+\Delta R)(C+\Delta C)} x_{2}+\frac{L \Delta C+\Delta L C+\Delta L \Delta C}{L C(L+\Delta L)(C+\Delta C)} x_{1}
\end{array}\right],
\end{aligned}
$$

where $\omega_{0}^{2}=1 / L C$.

Observed from (11), these lumped disturbances are caused by different disturbing factors, i.e., the circuit parameter vibrations such as $\Delta E, \Delta C$, and $\Delta L$ and the external 
disturbances such as $\Delta V_{\text {ref, }} \Delta R, d_{1}(t)$, and $d_{2}(t)$. And all the model uncertainties and external disturbances of buck converters are included. As mentioned above, much literature only considers one or several disturbances [10-13]. It is also the reason that diverse models have been established.

2.2. Analysis of the Multiple Lumped Disturbances. In the following, a unified modelling approach is proposed to cover all the possible disturbances as well as the possible situations with individual/several disturbances. Therefore, two cases are discussed.

Case 1. For (9), it contains all the model uncertainties and external disturbances. Here, we rewrite it as

$$
\dot{x}=\mathbf{A x}+[\mathbf{B}+\Delta \mathbf{B}] u+[\mathbf{D}+\zeta(\mathbf{x}, t)] .
$$

where $\zeta(x, t)=\Delta D_{1}+\Delta D_{2}(t)+\Delta D_{3}(x)$.

Case 2. All the individual disturbances of $\Delta E, \Delta C, \Delta L$, $\Delta R, \Delta V_{\text {ref }}, d_{1}(t)$, and $d_{2}(t)$ located in (9) are considered separately. Based on the modelling approach in (1) and Kirchhoff's circuit law, the corresponding average state-space models can be similarly deduced and summarized in Table 1 , where $\zeta_{i}(x, t)$ is the possible combination of the corresponding constant disturbance, state disturbance, and time-varying disturbance. The disturbance matrices $\Delta B_{j}, \Delta D_{1 i}$, and $\Delta D_{2 j}, i=1,2$, $j=1,2,3$, are deduced as

$$
\begin{aligned}
& \Delta \mathbf{B}_{1}=\left[\begin{array}{c}
0 \\
\frac{\Delta E}{L C}
\end{array}\right] \\
& \Delta \mathbf{B}_{2}=\left[\begin{array}{c}
0 \\
\frac{-\Delta C E}{L C(C+\Delta C)}
\end{array}\right] \text {, } \\
& \Delta \mathbf{B}_{3}=\left[\begin{array}{c}
0 \\
\frac{-\Delta L E}{L C(L+\Delta L)}
\end{array}\right] \text {, } \\
& \Delta \mathbf{D}_{11}(\mathbf{x})=\left[\begin{array}{c}
0 \\
\frac{R \Delta C V_{r e f}+L \Delta C x_{2}+R \Delta C x_{1}}{L C(C+\Delta C)}
\end{array}\right], \\
& \Delta \mathbf{D}_{12}(\mathbf{x})=\left[\begin{array}{c}
0 \\
\frac{\Delta L}{L C(L+\Delta L)} V_{\text {ref }}+\frac{\Delta L}{L C(L+\Delta L)} x_{1}
\end{array}\right] \text {, } \\
& \Delta \mathbf{D}_{21}(\mathbf{x})=\left[\begin{array}{c}
0 \\
\frac{\Delta R}{R C(R+\Delta R)} x_{2}
\end{array}\right] \text {, } \\
& \Delta \mathbf{D}_{22}=\left[\begin{array}{c}
0 \\
\frac{-\Delta V_{\text {ref }}}{L C}
\end{array}\right] \text {, } \\
& \Delta \mathbf{D}_{23}(t)=\left[\begin{array}{c}
0 \\
\dot{d}_{2}(t)
\end{array}\right] \text {. }
\end{aligned}
$$

By combining (12) and Table 1, here we further classify them into two cases. Because of coupling with the input control $u$, it is obvious that the input disturbance $\Delta B_{j}$ could hinder the controller design so that is categorized separately where $j=1,2,3$ while the others are classified into a group. Therefore, the two unified expressions in Table 1 can cover all the possible situations of buck converters with the individual or several or all disturbances in practice.

2.3. Discretization of the Buck Converter System. In the following, we adopt the zero-order holder $(\mathrm{ZOH})$ to realize the discretization of the buck converter system [24, 25, 27]. To cover the two unified expressions in Table 1, we take the worst situation with all the disturbances in (12) to discuss the system discretization.

By adopting $\mathrm{ZOH}$, the state value can be held constant during the sampling period $h$ until the next moment arrives. Therefore, the continuous model (12) is changed into a sampled-data system as

$$
\begin{aligned}
\mathbf{x}(k+1)= & \boldsymbol{\Phi} \mathbf{x}(k)+\Gamma \mathbf{B} u(k)+\Gamma \Delta \mathbf{B} u(k)+\Gamma\left[\mathbf{D}+\mathbf{D}_{1}\right. \\
& \left.+\Delta \mathbf{D}_{3}(\mathbf{x})\right]+\int_{0}^{h} e^{\mathbf{A} \tau}\left[\Delta \mathbf{D}_{2}(k+1) h-\tau\right] \mathrm{d} \tau
\end{aligned}
$$

where $\Phi$ and $\Gamma$ are denoted as

$$
\begin{gathered}
\boldsymbol{\Phi}=e^{\mathbf{A} h}=\mathbf{I}+\mathbf{A} h+\frac{\mathbf{A}^{2} h^{2}}{2 !}+O\left(h^{3}\right), \\
\Gamma=\int_{0}^{h} e^{\mathbf{A} \tau} \mathrm{d} \tau=h+\frac{h^{2} \mathbf{A}}{2 !}+O\left(h^{3}\right),
\end{gathered}
$$

where $I \in R^{2}$ and $O\left(h^{3}\right)$ is the item with orders higher than $h^{3}$ but ignored in this paper.

Furthermore, for (14), the time-varying disturbance $\left[\Delta D_{2}(k+1) h-\tau\right]$ and input disturbance $\Gamma \Delta \mathrm{Bu}(\mathrm{k})$ hinder the design of DTSM controller later. Therefore, they are needed to be dealt separately. For the former, based on the first integral mean value theorem [33], the time-varying disturbance $\left[\Delta D_{2}(k+1) h-\tau\right]$ is equivalent to

$$
\int_{0}^{h} e^{\mathbf{A} \tau} \Delta \mathbf{D}_{2}[(k+1) h-\tau] \mathrm{d} \tau=\int_{0}^{h} e^{\mathbf{A} \tau} \mathrm{d} \tau \Delta \mathbf{D}_{2}(\xi),
$$

where $\xi \in[k h,(k+1) h]$. While for the latter, the coupling of input disturbance $\Delta B$ and the control $u(k)$ still exist after discretization. Fortunately, due to the superiority of the direct ON/OFF control in Figure 1, the $\mathrm{ZOH}$ discretization of $u$ in (2) can be expressed as

$$
u(k)=-\frac{1}{2}\left(s_{k}-1\right),
$$

where $s_{k}=\operatorname{sgn}[s(k)]$. And if the power switch $S_{w}$ is OFF, there is $u(k)=0$ and $\Gamma \Delta B u(k)=0$ while on the contrary, it has $u(k)=1$ and $\Gamma \Delta B u(k)=\Gamma \Delta B$. Here, we take the worst case to cover the impact of the input disturbance $\Delta B$, i.e., replacing the disturbance term $\Gamma \Delta B u(k)$ in (14) by $\Gamma \Delta B$. 
Table 1: Average state-space models of buck converters with individual disturbances.

\begin{tabular}{|c|c|c|c|c|c|c|}
\hline \multirow{2}{*}{ Disturbing factors } & \multicolumn{4}{|c|}{ Lumped disturbances } & \multirow{2}{*}{ Average state-space model } & \multirow{2}{*}{ Unified expression } \\
\hline & $\Delta B$ & $\Delta D_{1}$ & $\Delta D_{2}(t)$ & $\Delta D_{3}(x)$ & & \\
\hline$\overline{\Delta \mathrm{E}}$ & $\sqrt{ }$ & & & & $\dot{x}=\mathbf{A x}+\left(\mathbf{B}+\Delta \mathbf{B}_{1}\right) u+\mathbf{D}$ & \\
\hline$\Delta \mathrm{C}$ & $\sqrt{ }$ & $\sqrt{ }$ & & $\sqrt{ }$ & $\dot{x}=\mathbf{A x}+\left(\mathbf{B}+\Delta \mathbf{B}_{2}\right) u+\mathbf{D}+\Delta \mathbf{D}_{11}(\mathbf{x})$ & $\dot{x}=\mathbf{A} \mathbf{x}+\left(\mathbf{B}+\Delta \mathbf{B}_{j}\right) u+\mathbf{D}+\zeta_{1}(\mathbf{x}, t)$ \\
\hline$\Delta \mathrm{L}$ & $\sqrt{ }$ & $\sqrt{ }$ & & $\sqrt{ }$ & $\dot{x}=\mathbf{A} \mathbf{x}+\left(\mathbf{B}+\Delta \mathbf{B}_{3}\right) u+\mathbf{D}+\Delta \mathbf{D}_{12}(\mathbf{x})$ & \\
\hline & & & & $\sqrt{ }$ & $\dot{x}=\mathbf{A x}+\mathbf{B} u+\mathbf{D}+\Delta \mathbf{D}_{21}(\mathbf{x})$ & \\
\hline $\begin{array}{l}\Delta \mathrm{V}_{\text {ref }} \\
d_{1}(t), d_{2}(t)\end{array}$ & & $\sqrt{ }$ & $\sqrt{ }$ & & $\begin{array}{c}\dot{x}=\mathbf{A} \mathbf{x}+\mathbf{B} u+\mathbf{D}+\Delta \mathbf{D}_{22} \\
\dot{x}=\mathbf{A x}+\mathbf{B} u+\mathbf{D}+\Delta \mathbf{D}_{23}(t)\end{array}$ & $\dot{x}=\mathbf{A} \mathbf{x}+\mathbf{B} u+\mathbf{D}+\zeta_{2}(\mathbf{x}, t)$ \\
\hline
\end{tabular}

By combining (14), (17), and (18), the discretization of the continuous model (12) can be finally obtained as

$$
\begin{aligned}
\mathbf{x}(k+1)= & \boldsymbol{\Phi} \mathbf{x}(k)+\Gamma \mathbf{B} u(k)+\Gamma \mathbf{D}+\Gamma\left[\Delta \mathbf{B}+\Delta \mathbf{D}_{1}\right. \\
& \left.+\Delta \mathbf{D}_{2}(\xi)+\Delta \mathbf{D}_{3}(\mathbf{x})\right] .
\end{aligned}
$$

Observed from (11), since the first rows of the matrix $\Delta B$, $\Delta D_{1}, \Delta D_{2}(\xi)$, and $\Delta D_{3}(x)$ are all zero, we define $\Delta B+\Delta D_{1}+\Delta D_{2}(\xi)+\Delta D_{3}(x)=D_{4}(x, \xi)=\left[0, d_{3}(x, \xi)\right]^{T}$, where

$$
\begin{aligned}
d_{3}(\mathbf{x}, \xi)= & \frac{\Delta E L C-L C \Delta V_{\mathrm{ref}}}{L C(L+\Delta L)(C+\Delta C)}+\frac{\left(x_{1}-E+V_{\mathrm{ref}}\right)}{L C} \\
& -\frac{\left(x_{1}-E+V_{\mathrm{ref}}\right) L C}{L C(L+\Delta L)(C+\Delta C)}+\frac{d_{1}(t)}{(C+\Delta C)}+\dot{d}_{2}(t) \\
& +\frac{x_{2}}{R C}-\frac{R C}{R C(R+\Delta R)(C+\Delta C)} x_{2} .
\end{aligned}
$$

It is worth noticing that the discrete model in (19) corresponds to the worse situation with all the disturbances in (12). From Table 1, the difference of the two unified models of buck converters lies in the term $\Delta B_{j} u, j=1,2,3$. Therefore, the discrete models of the two unified expressions can be similarly deduced as

$$
\left\{\begin{array}{l}
\mathbf{x}(k+1)=\boldsymbol{\Phi} \mathbf{x}(k)+\Gamma \mathbf{B} u(k)+\Gamma \mathbf{D}+\Gamma\left[\Delta \mathbf{B}_{j}+\zeta_{1}(\mathbf{x}, \xi)\right] \\
\mathbf{x}(k+1)=\boldsymbol{\Phi} \mathbf{x}(k)+\Gamma \mathbf{B} u(k)+\Gamma \mathbf{D}+\Gamma \zeta_{2}(\mathbf{x}, \xi)
\end{array}\right.
$$

where $\xi \in[k h,(k+1) h]$ is the same as $(17)$ and $\zeta_{i}(x, \xi)$ corresponds to the discretization of the disturbances $\zeta_{i}(x, t)$, $i=1,2$.

From (13), since $\zeta_{i}(x, \xi)$ represents the combination of the possible constant disturbance, state disturbance, and time-varying disturbance, they can be further denoted as $d_{3 i}(x, \xi)$ as $(20)$, where $i=1,2$. In essence, the two discrete models in (21) can be concluded into a unified expression as
(19) despite of different disturbing terms, which depends on the contained disturbances in practice. It also proves the effectiveness of the two unified expressions in Table 1, which can cover all the possible situations of buck converters with the individual or several or all disturbances in practice.

\section{Robust Stability of DTSM Controlled Buck Converters}

In the following, a unified DTSM control approach is proposed based on the above unified modelling and analysis of the robust stability for buck converters. Still taking the worse situation with all the disturbances in (19) as an example, the influences of the sampling time and lumped disturbances on the control system are two key problems need to be addressed.

3.1. Design of DTSM Controller. Due to the advantage of easy implementation, the discrete signals $x_{1}(k)$ and $x_{2}(k)$ are adopted to design a linear SM surface $s(k)$ for the ON/OFF control of buck converter in Figure 1 as

$$
s(k)=\lambda x_{1}(k)+x_{2}(k)=\mathbf{c x}(k),
$$

where the design parameter $c=[\lambda, 1], \lambda>0$. In fact, other types of sliding surfaces such as terminal SM and nonsingular terminal SM [19] can also be used, wherein the sliding variable $s(k)$ can be regarded as a threshold in (18) to trigger the power switch.

Theorem 1 (robust stability analysis). For the discrete model of buck converter in (19), if the sliding surface $s(k)$ is designed as (22), the control law $u(k)$ is implemented as (18) and the robust stability condition is satisfied with the following inequalities, where $l_{1}(\lambda, h)$ and $l_{2}(\lambda, h)$ are defined to express the corresponding conditions when the power switch is $O N$ and OFF, respectively, as follows:

$$
\left\{\begin{array}{l}
l_{1}(\lambda, h)=-\left(\omega_{0}^{2}+\frac{\lambda \omega_{0}^{2} h}{2}-\frac{\omega_{0}^{2} h}{2 R C}\right) x_{1}(k)+\left[\lambda-\frac{1}{R C}+\left(\frac{1}{R^{2} C^{2}}-\frac{\lambda}{R C}-\omega_{0}^{2}\right) \frac{h}{2}\right] x_{2}(k)-\left(1+\frac{\lambda h}{2}-\frac{h}{2 R C}\right)\left[V_{\text {ref }} \omega_{0}^{2}-d_{3}(x, \xi)\right]<0, \\
l_{2}(\lambda, h)=-\left(\omega_{0}^{2}+\frac{\lambda \omega_{0}^{2} h}{2}-\frac{\omega_{0}^{2} h}{2 R C}\right) x_{1}(k)+\left[\lambda-\frac{1}{R C}+\left(\frac{1}{R^{2} C^{2}}-\frac{\lambda}{R C}-\omega_{0}^{2}\right) \frac{h}{2}\right] x_{2}(k)+\left(1+\frac{\lambda h}{2}-\frac{h}{2 R C}\right)\left[\omega_{0}^{2}\left(E-V_{\text {ref }}\right)+d_{3}(x, \xi)\right]>0, \quad \text { for } s(k)<0, u(k)=1 .
\end{array}\right.
$$


Then, the whole closed-loop DTSM control system can be guaranteed stable.

Proof. In this paper, differing from the traditional DTSM approach based on the nominal model [1, 24, 27, 31], since all the possible model uncertainties and external disturbances in (19) are included into the controller design, the existing condition of DTSM should be satisfied [24, 29-32], i.e.,

$$
s^{2}(k+1)<s^{2}(k),
$$

which is equivalent to $|s(k+1)|<|s(k)|$.

By substituting (18) into (19), the whole closed-loop DTSM control system can be obtained as

$$
\mathbf{x}(k+1)=\boldsymbol{\Phi} \mathbf{x}(k)-\frac{1}{2} \Gamma \mathbf{B} s_{k}+\mathbf{v}(k)
$$

where the total lumped disturbance $v(k)$ is denoted as

$$
\mathbf{v}(k)=\Gamma\left[\frac{1}{2} \mathbf{B}+\mathbf{D}+\mathbf{D}_{4}(\mathbf{x}, \xi)\right] .
$$

From (15) and (16), $s(k+1)$ can be further obtained from (22) as

$$
\begin{aligned}
s(k+1)= & \mathbf{c x}(k)+(\mathbf{c} \Phi-\mathbf{c}) \mathbf{x}(k) \\
& +\mathbf{c v}(k)-\frac{1}{2} \mathbf{c} \Gamma \mathbf{B} s_{k}=s(k)+\Psi(k)-\boldsymbol{\Theta} s_{k},
\end{aligned}
$$

where the variables $\psi(k)$ and $\Theta$ can be deduced as

$$
\begin{aligned}
\Psi(k) & =(\mathbf{c} \boldsymbol{\Phi}-\mathbf{c}) \mathbf{x}(k)+\mathbf{c v}(k), \\
\boldsymbol{\Theta} & =\frac{1}{2} \mathbf{c} \Gamma \mathbf{B} .
\end{aligned}
$$

Based on the existing condition of DTSM in (24), two cases in accordance with the power switch ON/OFF need to be discussed in the following:

(1) If $s(k)>0$, then $u(\mathrm{k})=0$ can be obtained from (18). And the existing condition of DTSM in (24) is equivalent to $s(k+1)<s(k)$ for the case $s(k+1)>0$ and $s(k+1)>s(k)$ for the other case $s(k+1)<0$. However, as the system converges to the equilibrium point in the phase plane $\left(x_{1}, x_{2}\right)$, i.e., $\left(\left(x_{1}(k)=0\right.\right.$, $\left.\left.x_{2}(k)=0\right)\right)$, it is easy to prove that the condition $s(k)$ $s(k+1)<0$ will make the system run across the sliding line $s(k)=0$ and cannot be guaranteed to always hold [34]. Therefore, from (27)-(29), the guaranteed robust stability condition can be obtained as

$$
(\mathbf{c} \Phi-\mathbf{c}) \mathbf{x}(k)+\mathbf{c v}(k)-\frac{1}{2} \mathbf{c} \Gamma \mathbf{B}<0 .
$$

(2) If $s(k)<0$, similarly $s(k+1)>s(k), u(k)=1$ holds and the stability condition is deduced as

$$
(\mathbf{c} \Phi-\mathbf{c}) \mathbf{x}(k)+\mathbf{c v}(k)+\frac{1}{2} \mathbf{c} \Gamma \mathbf{B}>0 .
$$

Furthermore, by substituting (15), (16), and (20) into (30) and (31), the robust stability condition in cases of power switch ON and OFF can be changed as

$$
\begin{cases}\mathbf{c}\left(\mathbf{A} h+\frac{\mathbf{A}^{2} h^{2}}{2}\right) \mathbf{x}(k)+\mathbf{c}\left(h \mathbf{I}+\frac{\mathbf{A} h^{2}}{2}\right)\left[\mathbf{D}+\mathbf{D}_{4}(\mathbf{x}, \xi)\right]<0, & \text { for } s(k)>0, u(k)=0, \\ \mathbf{c}\left(\mathbf{A} h+\frac{\mathbf{A}^{2} h^{2}}{2 !}\right) \mathbf{x}(k)+\mathbf{c}\left(h \mathbf{I}+\frac{h^{2} \mathbf{A}}{2}\right)\left[\mathbf{B}+\mathbf{D}+\mathbf{D}_{4}(\mathbf{x}, \xi)\right]>0, & \text { for } s(k)<0, u(k)=1 .\end{cases}
$$

For the items $c\left(A h+A^{2} h^{2} / 2\right)$ and $c\left(h I+A h^{2} / 2\right)$ in (32), they can be further denoted from (10) as

$$
\begin{aligned}
& \mathbf{c}\left(\mathbf{A} h+\frac{\mathbf{A}^{2} h^{2}}{2}\right)=\left[\begin{array}{c}
-\omega_{0}^{2} h-\frac{1}{2} \lambda \omega_{0}^{2} h^{2}+\frac{1}{2} \frac{\omega_{0}^{2} h^{2}}{R C} \\
-\frac{h}{R C}+\frac{1}{2}\left(-\omega_{0}^{2}+\frac{1}{R^{2} C^{2}}\right) h^{2}+\lambda\left(h-\frac{h^{2}}{2 R C}\right)
\end{array}\right]^{T}, \\
& \mathbf{c}\left(h \mathbf{I}+\frac{\mathbf{A} h^{2}}{2}\right)=\left[\begin{array}{c}
\lambda h-\frac{\omega_{0}^{2} h}{2} \\
h+\frac{\lambda h^{2}}{2}-\frac{h^{2}}{2 R C}
\end{array}\right]^{T} .
\end{aligned}
$$


Therefore, by substituting (33) and (34) into (32), the robust stability condition (23) can be finally achieved.

3.2. Influence of the Sampling Time on Controller Regulation. From the guaranteed stability condition in Theorem 1 and (23), it is known that the control performance of the system is determined by the sampling time $h$, lumped disturbance $d_{3}(x, \xi)$, and DTSM parameter $\lambda$ simultaneously. In the following, we first investigate the relationship of the sampling time $h$ and DTSM parameter $\lambda$ in the phase plane $\left(x_{1}\right.$, $\left.x_{2}\right)$.

Theorem 2 (relationship of the sampling time and DTSM parameter). In order to guarantee the robust stability of DTSM controlled buck converter system in (25), the parameter $\lambda$ is recommended to be chosen within the four subranges: (1) $0<\lambda<\psi_{1}$; (2) $\psi_{1}<\lambda<\psi_{2}$; (3) $\psi_{2}<\lambda<\psi_{3}$; and (4) $\lambda>\psi_{3}$, where

$$
\left\{\begin{array}{l}
\psi_{1}=\frac{1}{R C}-\frac{2}{h}, \\
\psi_{2}=\frac{1}{R C}, \\
\psi_{3}=\frac{(2 / R C)+\omega_{0}^{2} h-\left(1 / h R^{2} C^{2}\right)}{2-(h / R C)} .
\end{array}\right.
$$

Proof. Based on the guaranteed stability condition in (23), the slopes of the lines $l_{1}(\lambda, h)$ and $l_{2}(\lambda, h)$ are the same and denoted as

$$
\rho(\lambda, h)=\frac{[1+(\lambda-1 / R C) h / 2] \omega_{0}^{2}}{(\lambda-(1 / R C))-0.5\left(\omega_{0}^{2}-\left(1 / R^{2} C^{2}\right)+(\lambda / R C)\right) h} .
$$

For the comparative study imposed by the lumped disturbance $d_{3}(x, \xi)$, the stability condition of the nominal counterpart of (19) can also be deduced as

$$
\left\{\begin{array}{l}
l_{1}^{\prime}(\lambda, h)=-\left(\omega_{0}^{2}+\frac{\lambda \omega_{0}^{2} h}{2}-\frac{\omega_{0}^{2} h}{2 R C}\right) x_{1}(k)+\left[\lambda-\frac{1}{R C}+\left(\frac{1}{R^{2} C^{2}}-\frac{\lambda}{R C}-\omega_{0}^{2}\right) \frac{h}{2}\right] x_{2}(k)-V_{\text {ref }} \omega_{0}^{2}\left(1+\frac{\lambda h}{2}-\frac{h}{2 R C}\right)<0, \\
l_{2}^{\prime}(\lambda, h)=-\left(\omega_{0}^{2}+\frac{\lambda \omega_{0}^{2} h}{2}-\frac{\omega_{0}^{2} h}{2 R C}\right) x_{1}(k)+\left[\lambda-\frac{1}{R C}+\left(\frac{1}{R^{2} C^{2}}-\frac{\lambda}{R C}-\omega_{0}^{2}\right) \frac{h}{2}\right] x_{2}(k)+\omega_{0}^{2}\left(1+\frac{\lambda h}{2}-\frac{h}{2 R C}\right)\left(E-V_{\text {ref }}\right)>0, \quad \text { for } s(k)<0, u(k)=1,
\end{array}\right.
$$

where $l_{1}^{\prime}(\lambda, h)$ and $l_{1}^{\prime}(\lambda, h)$ are defined to express the corresponding conditions when the power switch is $\mathrm{ON}$ and OFF, respectively. It is worth noticing that their slopes are as the same as $\rho(\lambda, h)$ in (36).

Here, we define two variables $h_{1}(\lambda)$ and $h_{2}(\lambda)$ as

$$
\left\{\begin{array}{l}
h_{1}(\lambda)=\frac{2(\lambda-(1 / R C))}{\omega_{0}^{2}+(\lambda / R C)-\left(\lambda / R^{2} C^{2}\right)} \\
h_{2}(\lambda)=-\frac{2}{\lambda-(1 / R C)}
\end{array}\right.
$$

which are the two critical values of the denominator and numerator, which determine the sign of the slope $\rho(\lambda, h)$ in (36).

According to the ON/OFF operation of the power switch, as well as the two critical variables $h_{1}(\lambda)$ and $h_{2}(\lambda)$ in (38), the robust stability conditions of the DTSM controlled buck converter system with/without disturbances, i.e., (23) and (37) are illustrated and compared in Figures 2(a)-2(c), where these regions are mutually intersected by the sliding line $s(k)=0$ and the lines $l_{1}(\lambda, h)$ and $l_{2}(\lambda, h)$. The four regions are defined as follows: Region I $\left(l_{1}(\lambda, h)>0, s(k)<0\right.$, and $u=1)$; Region $\operatorname{II}\left(l_{2}(\lambda, h)<0, s(k)>0\right.$, and $\left.u=0\right)$; Region III $\left(l_{1}^{\prime}(\lambda, h), s(k)<0\right.$, and $\left.u=1\right)$; and Region IV $\left(l_{2}^{\prime}(\lambda, h)<0\right.$, $s(k)>0$, and $u=0)$. It should be noted that the lines $l_{1}(\lambda, h)$ and $l_{2}(\lambda, h)$ may not be straight lines due to the existence of the time-varying disturbance $d_{3}(x, \xi)$. In phase plane $\left(x_{1}, x_{2}\right)$, four cases will be discussed in the following.

Case 1. $\rho(\lambda, h)<0$ : For the two terms $(\lambda-1 / R C)$ and $(\lambda-1 / \mathrm{RC})-0.5\left(\omega_{0}^{2}-1 / \mathrm{R}^{2} \mathrm{C}^{2}+\lambda / R C\right) h$ located in the denominator of $\rho(\lambda, h)$ in (36), if $(\lambda-1 / \mathrm{RC})>0$, then $h_{2}(\lambda)<0$ in (38) always holds. Furthermore, if $h_{1}(\lambda)<h$, it has $(\lambda-1 / R C)-0.5\left(\omega_{0}^{2}-1 / R^{2} C^{2}+\lambda / R C\right) h<0$, the denominator of $\rho(\lambda, h)$ will be positive, and the slope $\rho(\lambda, h)<0$ holds for all lines $l_{1}(\lambda, h), l_{2}(\lambda, h), l_{1}^{\prime}(\lambda, h)$, and $l_{2}^{\prime}(\lambda, \mathrm{h})$ in (23) and (37) simultaneously. Therefore, by combining the conditions $(\lambda-1 / R C)>0$ and $(\lambda-1 /$ 


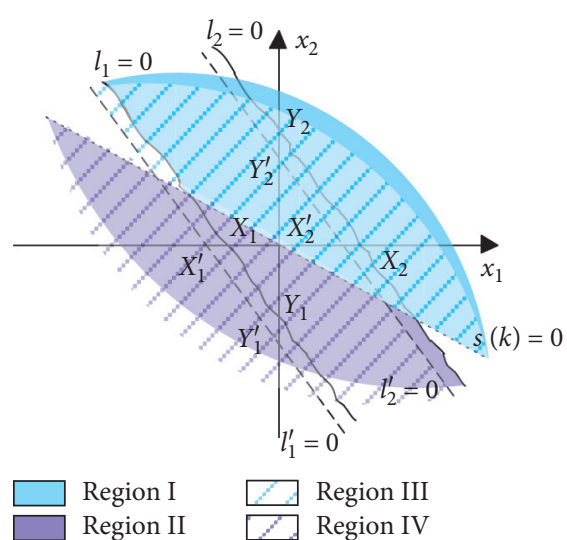

(a)

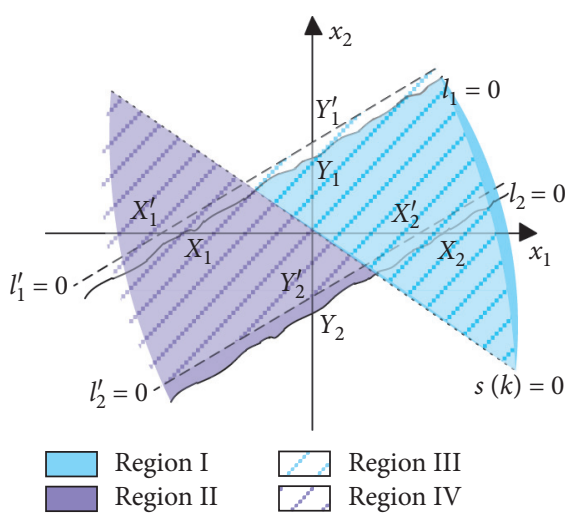

(b)
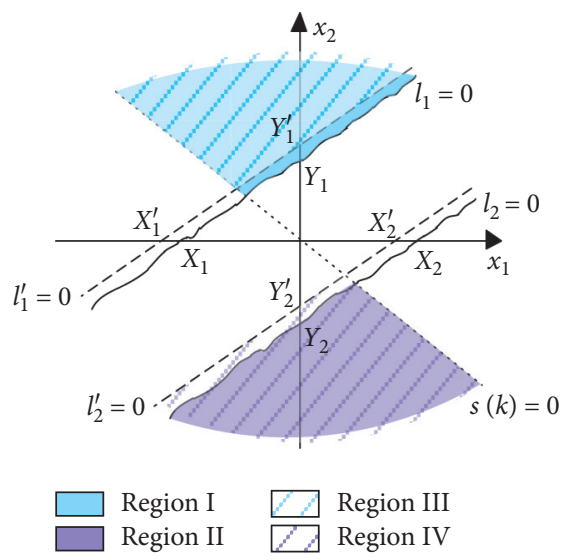

(c)

FiguRE 2: Robust stability regions: (a) $\rho(\lambda, h)<0$; (b) $\rho(\lambda, h)>0$ and $(\lambda-1 / \mathrm{RC})-0.5\left(\omega_{0}^{2}-1 / R^{2} C^{2}+\lambda / R C\right) h>0$; (c) $\rho(\lambda, h)>0$ and $[1+(\lambda-1 /$ RC) $h / 2] \omega_{0}^{2}<0$.

$R C)-0.5\left(\omega_{0}^{2}-1 / R^{2} C^{2}+\lambda / R C\right) h<0$, the robust stability regions are illustrated in Figure 2(a). Meanwhile, DTSM parameter $\lambda$ can be deduced as

$$
\frac{1}{R C}<\lambda<\frac{(2 / R C)+\omega_{0}^{2} h-\left(1 / h R^{2} C^{2}\right)}{2-(h / R C)} .
$$

Case 2. $\rho(\lambda, h)>0$ and $(\lambda-1 / \mathrm{RC})-0.5\left(\omega_{0}-1 / R^{2} C^{2}+\lambda /\right.$ $R C) h>0$ : Similarly, as per the analysis in Case 1 , if the two conditions $(\lambda-1 / \mathrm{RC})>0$ and $(\lambda-1 / R C)-$ $0.5\left(\omega_{0}^{2}-1 / R^{2} C^{2}+\lambda / R C\right) h>0$ are satisfied at the same time, it has $h_{1}(\lambda)>h, h_{2}(\lambda)<0$ in (38) and the slope $\rho(\lambda$, $h)>0$ in (36). Correspondingly, the robust stability regions are illustrated in Figure 2(b). And DTSM parameter $\lambda$ is

$$
\lambda>\frac{(2 / R C)+\omega_{0}^{2} h-\left(1 / h R^{2} C^{2}\right)}{2-(h / R C)} .
$$

Case 3. $\rho(\lambda, h)>0$ : Observed from (38), if $(\lambda-1 / R C)<0$, the variable $h_{1}(\lambda)<0$ always holds. To guarantee the sampling time $h>h_{2}(\lambda)$, it has $[1+(\lambda-1 / R C) h / 2] \omega_{0}^{2}<0$ and the numerator of $\rho(\lambda, h)$ is negative. Therefore, the slope $\rho(\lambda, h)>0$, and the robust stability regions are illustrated in Figure 2(c). And the DTSM parameter $\lambda$ is recommended as

$$
0<\lambda<\frac{1}{R C}-\frac{2}{h}
$$

Case 4. $\rho(\lambda, h)<0$ : Still let the variable $h_{1}(\lambda)<0$ as Case 3 based on the condition $(\lambda-1 / R C)<0$. In order to make the other variable $h<h_{2}(\lambda), \rho(\lambda, h)<0$ should be held so that the robust stability regions can be described as the same as Figure 2(a). Correspondingly, the DTSM parameter $\lambda$ is recommended as

$$
\frac{1}{R C}-\frac{2}{h}<\lambda<\frac{1}{R C}
$$

Based on the robust stability analysis of the above four cases, the relationship of the sampling time $h$ and DTSM parameter $\lambda$ can be determined. And accordingly, the three critical values of $\lambda$ can be finally obtained as (35).

Remark 1. Differing from the traditional DTSM based on the nominal model $[1,24,27,31]$, the disturbances are directly introduced in the controller design, giving four new subranges, shown in Figure 3. It is more accurate and timesaving.

Remark 2. For the recommended choice of DTSM parameter $\lambda$ in (35), in essence it is deduced from $\rho(\lambda, h)$ in (36), which is the slope of the lines $l_{1}(\lambda, h), l_{2}(\lambda, h), l_{1}^{\prime}(\lambda, h)$, and $l_{2}^{\prime}(\lambda, h)$ at the same time. In other words, the proposed design approach of DTSM controller in Theorem 2 is suitable for both the nominal and perturbed systems. It is also the reason of the so-called "unified" controller design in this paper.

Remark 3. Considering the limitation of the hardware circuit, special attention should be paid to the subrange $0<\lambda<\psi_{1}$, where the sampling time is restricted with $h>2 R C$. Meanwhile, it is known that the biggest switch frequency of power switch $f_{\mathrm{s}}$ is expected to be less than half of sampling frequency based on the Shannon sampling theorem [35], i.e., $f_{\mathrm{s}}<1 / 4 R C$. However, considering the switching capability of the physical power switch, e.g., IGBT and MOSFET, if the conditions $h>2 R C$ and $f_{s}<1 / 4 R C$ cannot be satisfied at the same time, the value of $\psi_{1}$ in (35) is of no practical use. In other words, (1) $0<\lambda<\psi_{2}$, (2) $\psi_{2}<\lambda<\psi_{3}$, and (3) $\lambda>\psi_{3}$ can be used to replace the four subranges in Theorem 2 .

3.3. Influence of the Lumped Disturbances on Control Performance. For the lumped disturbance $d_{3}(x, \xi)$ in (19), since it is directly contained in the design of DTSM controller, it does not affect the system stability. Guaranteed by 


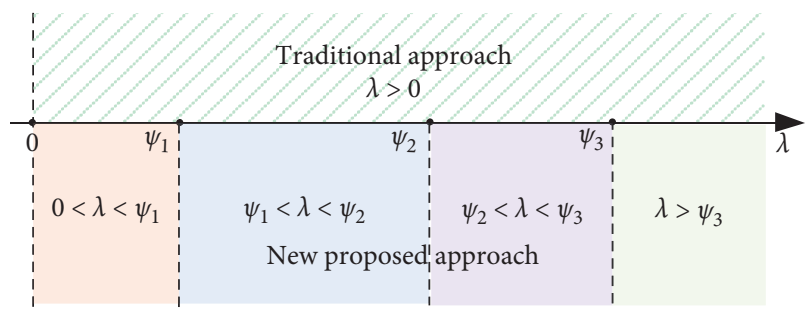

FIGURE 3: Difference of the traditional and proposed DTSM parameter.

the robust stability condition in (23) while from the comparisons of the DTSM controlled buck converter with/ without disturbances in Figures 2(a)-2(c), it will affect the steady-state error of the system, which can be estimated from the boundary of the robust stability regions. Therefore, how the lumped disturbance $d_{3}(x, \xi)$ affects the control performance is another important question need to be addressed.
Theorem 3 (influence of the lumped disturbance on the steady-state error). For the DTSM controlled buck converter in (25), if the robust stability condition (23) is satisfied and the DTSM parameter $\lambda$ is chosen as (35), the output voltage $v_{c}$ varies within $\left[d_{3}(x, \xi) / \omega_{0}^{2}, E+d_{3}(x, \xi) / \omega_{0}^{2}\right]$ and the error of its changing rate is $d_{3}(x, \xi) /(\lambda-1 / R C)$.

Proof. For the DTSM controlled buck converter in (25), it can be stabilized under the robust stability condition (23), i.e., after the sliding variable $s(k)$ in $(22)$ is forced to zero, the equilibrium point of the system in phase plane $\left(x_{1}, x_{2}\right)$ can be calculated as $(0,0)$. From (1), since the output voltage $v_{c}(k)=x_{1}(k)-V_{\text {ref }}$ and $\dot{v}_{c}(k)=x_{2}(k)$, their steady-state errors can be estimated from the boundary of the robust stability regions in Figures 2(a)-2(c).

In Region I and Region II with lumped disturbances $d_{3}(x, \xi)$, the size of the boundary is determined by the four intersection points of $l_{1}(\lambda, h)$ and $l_{2}(\lambda, h)$ with $x_{1}$ axis and $x_{2}$ axis, i.e., $X_{1}(k), X_{2}(k), Y_{1}(k)$, and $Y_{2}(k)$. Therefore, we can calculate them from (23) and (36) as

$$
\left\{\begin{array}{l}
X_{1}(k)=\left(-V_{\mathrm{ref}}+\frac{d_{3}(\mathbf{x}, \zeta)}{\omega_{0}^{2}}, 0\right), \\
X_{2}(k)=\left(E-V_{\mathrm{ref}}+\frac{d_{3}(\mathbf{x}, \zeta)}{\omega_{0}^{2}}, 0\right) \\
Y_{1}(k, \lambda, h)=\left(0, \frac{-[1+0.5(\lambda-(1 / R C)) h]\left[\omega_{0}^{2} V_{\mathrm{ref}}-d_{3}(\mathbf{x}, \xi)\right]}{\left[-(\lambda-(1 / R C))+0.5\left(\omega_{0}^{2}-\left(1 / R^{2} C^{2}\right)+(\lambda / R C)\right) h\right]}\right) \\
Y_{2}(k, \lambda, h)=\left(0, \frac{-[1+0.5(\lambda-(1 / R C)) h]\left[\omega_{0}^{2}\left(E-V_{\mathrm{ref}}\right)+d_{3}(\mathbf{x}, \xi)\right]}{\left[-(\lambda-(1 / R C))+0.5\left(\omega_{0}^{2}-\left(1 / R^{2} C^{2}\right)+(\lambda / R C)\right) h\right]}\right)
\end{array}\right.
$$

While in Region III and Region IV without lumped disturbances (nominal system), the four points of $l_{1}^{\prime}(\lambda, h)$ and $l_{2}^{\prime}(\lambda, h)$ with $x_{1}$ axis and $x_{2}$ can be obtained from (37) as

$$
\left\{\begin{array}{l}
X_{1}^{\prime}(k)=\left(-V_{\text {ref }}, 0\right) \\
X_{2}^{\prime}(k)=\left(E-V_{\text {ref }}, 0\right), \\
Y_{1}^{\prime}(k, \lambda, h)=\left(0, \frac{-\omega_{0}^{2} V_{\text {ref }}[1+0.5(\lambda-(1 / R C)) h]}{\left[-(\lambda-(1 / R C))+0.5\left(\omega_{0}^{2}-\left(1 / R^{2} C^{2}\right)+(\lambda / R C)\right) h\right]}\right), \\
Y_{2}^{\prime}(k, \lambda, h)=\left(0, \frac{-\omega_{0}^{2} V_{\text {ref }}[1+0.5(\lambda-(1 / R C)) h]}{\left[-(\lambda-(1 / R C))+0.5\left(\omega_{0}^{2}-\left(1 / R^{2} C^{2}\right)+(\lambda / R C)\right) h\right]}\right) .
\end{array}\right.
$$


By comparing the four points $X_{1}(k)$ and $X_{2}(k)$ in (43) and $X_{1}^{\prime} 1(k)$ and $X_{2}^{\prime}(k)$ in $(44)$, we can see that, as the reference output voltage $V_{\text {ref }}$ varies within $[0, E]$, the output voltage varies within $\left[d_{3}(x, \xi) / \omega_{0}^{2}, E+d_{3}(x, \xi) / \omega_{0}^{2}\right]$.

If we further let $h \longrightarrow 0$, the changing rate of the output voltage $\dot{v}_{c}(k)$ can be further estimated from the four points $Y_{1}(k, \lambda, h), Y_{1}^{\prime}(k, \lambda, h), Y_{2}(k, \lambda, h)$, and $Y_{2}^{\prime}(k, \lambda, h)$, where

$$
\begin{aligned}
& \left\{\begin{array}{l}
Y_{1}(k, \lambda, 0)=\left(0, \frac{\omega_{0}^{2} V_{r e f}-d_{3}(\mathbf{x}, \xi)}{\lambda-(1 / R C)}\right), \\
Y_{1}^{\prime}(k, \lambda, 0)=\left(0, \frac{\omega_{0}^{2} V_{\text {ref }}}{\lambda-(1 / R C)}\right)
\end{array}\right. \\
& \left\{\begin{array}{l}
Y_{2}(k, \lambda, 0)=\left(0,-\frac{\omega_{0}^{2}\left(E-V_{\mathrm{ref}}\right)+d_{3}(\mathbf{x}, \xi)}{\lambda-(1 / R C)}\right), \\
Y_{2}^{\prime}(k, \lambda, 0)=\left(0,-\frac{\omega_{0}^{2}\left(E-V_{\mathrm{ref}}\right)}{\lambda-(1 / R C)}\right) .
\end{array}\right.
\end{aligned}
$$

By comparing (45) and (46), it can be seen that the changing rate of output voltage $\dot{v}_{c}(k)$ is also affected by the lumped disturbance $d_{3}(x, \xi)$ and the error of its changing rate can be obtained as $d_{3}(x, \xi) /(\lambda-1 / R C)$. In other words, the disturbance $d_{3}(x, \xi)$ imposes a parallel shift, shown in Figures 2(a)-2(c).

\section{Simulations and Experiments}

In order to validate the unified approach of modelling and controller design for the buck converter in Figure 1, its circuit parameters are given as follows: $E=18 \mathrm{~V}$, $L=1000 \mu \mathrm{H}, C=3200 \mu \mathrm{F}, R=10 \Omega$, and $V_{\text {ref }}=9 \mathrm{~V}$.

4.1. Simulation Results. Based on the results of Theorems $1-3$, the proposed unified design approach of the DTSM controller can be concluded as the following two steps:

Step 1. Regardless of the buck converter with one or several or the all disturbances in practice, it is recommended to ignore its disturbance temporarily and to choose DTSM parameter $\lambda$ and sampling time $h$. In other words, the practical model with disturbance is replaced by its nominal counterpart and further applied (35) to it.

Based on the relationship of the sampling time $h$ and DTSM parameter $\lambda$ in (35), it has $\psi_{2}=1 / R C=31.25$, and $\psi_{1}$ and $\psi_{3}$ are determined by the sampling time $h$. Based on the Shannon sampling theorem [35], the biggest switch frequency of power switch $f_{s}$ is expected to be less than half of sampling frequency, i.e., $f_{s}<1 / 4 R C$ $(78.1 \mathrm{~Hz})$ and the sampling time $h$ is expected with $h>2 R C(6.4 \mathrm{~ms})$. Since the switching frequency of the physical IGBT and MOSFET used in power converters is general dozens of $\mathrm{kHz}$, the four subranges in Figure 3 can be replaced by the following three subranges: (1) $0<\lambda<31.25$, (2) $31.25<\lambda<\psi_{3}$, and (3) $\lambda>\psi_{3}$. In this paper, we choose the sampling time $h=1 \mathrm{~ms}, 0.5 \mathrm{~ms}$, and $0.25 \mathrm{~ms}$ for comparisons and then $\psi_{3}=189.98$, 109.99 , and 70.47, respectively. Correspondingly from (35), DTSM parameter $\lambda$ can be chosen as 15,60 , and 250 , respectively. The performance comparisons with different pairs of $(h, \lambda)$ are shown in Figure 4 and Table 2 .

In Figure 4 , by comparing the output voltages $v_{c}$ labeled from $A 1$ to $A 9$, it can be seen that the control performances of the buck converter are affected by the sampling time $h$ and DTSM parameter $\lambda$ simultaneously. From Table 2 , the nine pairs of $(h, \lambda)$ are all suitable in practical applications. Especially for $A 1$, $A 2$, and $A 3$ with the same sampling time $h=1 \mathrm{~ms}$, their response time, steady error, and relative steady error of output voltages $v_{c}$ are almost the same, listed as $4 \mathrm{~ms}, 3.90$, and $0.434 \%$, respectively. It means that the choice of the pair $(h, \lambda)$ is not unique for the same performance index. Continuously, we compare $A 4$, $A 5$, and $A 6$ with $h=0.5 \mathrm{~ms}$ and $A 7, A 8$, and $A 9$ with $\bar{h}=0.25 \mathrm{~ms}$ from two aspects. On the one side, if we fix the sampling time $h$, the bigger the DTSM parameter $\lambda$ is, the smaller the response time is, while the values of steady error and the relative steady error of the output voltages $v_{c}$ are maintained. It means that the sampling time $h$ is more sensitive to the system than the DTSM parameter $\lambda$. Therefore, it is recommended to determine the value of $h$ prior to that of $\lambda$. On the other side, if we fix the DTSM parameter $\lambda$, with the decrease in the sampling time $h$, the values of the response time will increase inversely, while the values of the steady error and $v_{c}$ will decrease. Since the regulation of the DTSM controller is based on the stability condition in (23), the deduced pair $(h, \lambda)$ in (35) only affects the static performances (e.g., steady error and relative steady error) but is not directly related to the dynamic performance (e.g., response time), which is listed in Table 2 just for reference.

Step 2: For the bounded lumped disturbance $d_{3}(x, \xi)$ in (20), the next is to determine its influences on the system. In order to cover all the possible model uncertainties and external disturbances in (11), here we assume $\Delta B=[0, \quad 0.0025 E \sin (2 \pi / h) / L C]^{T}, \quad \Delta D_{1}=[0$, $\left.0.0025 V_{\text {ref }} \sin (2 \pi / h)\right]^{T}, \Delta D_{2}(t)=\left[0,0.0025 V_{\text {ref }} \sin (2 \pi /\right.$

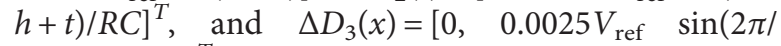
$\left.\left.h+x_{1} x_{2}\right) / R L\right]^{T}$. The reason of choosing the same amplitude $0.0025 V_{\text {ref }}$ for $\Delta D_{1}, \Delta D_{2}(t)$, and $D_{3}(x)$ is only to check whether the parallel shift function imposed by different disturbances is true or not. The sampling time is fixed as $h=0.5 \mathrm{~ms}$, and the DTSM parameter $\lambda$ is still chosen as 15,60 , and 250, respectively. The simulation results are given in Figure 5 and Table 2, labeled from $B 1$ to $B 3$.

In Figure 5(a) and Table 2, A4, A5, and A6 correspond to the nominal system, while $B 1, B 2$, and $B 3$ correspond to the perturbed system. By comparisons, it can be seen that their 


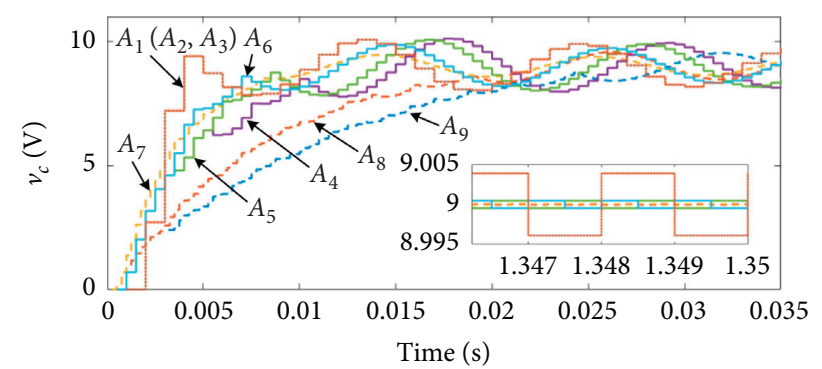

FIGURE 4: Comparisons of the output voltage with nine pairs of $(h, \lambda)$.

TABle 2: Performance comparisons with nine pairs of $(h, \lambda)$.

\begin{tabular}{|c|c|c|c|c|c|}
\hline$h$ & $\Lambda$ & Label & Response time (ms) & Steady error $(\mathrm{mV})$ & Relative steady error \\
\hline \multirow{3}{*}{$1 \mathrm{~ms}$} & 15 & $A 1$ & 4 & 3.902 & $0.434 \%$ o \\
\hline & 60 & $A 2$ & 4 & 3.902 & $0.434 \%$ \\
\hline & 250 & A3 & 4 & 3.902 & $0.434 \%$ o \\
\hline \multirow{3}{*}{$0.5 \mathrm{~ms}$} & 15 & $A 4$ & 9.5 & 0.465 & $0.052 \%$ o \\
\hline & 60 & $A 5$ & 8 & 0.465 & $0.052 \%$ o \\
\hline & 250 & $A 6$ & 6.5 & 0.465 & $0.052 \%$ o \\
\hline \multirow{3}{*}{$0.25 \mathrm{~ms}$} & 15 & $A 7$ & 20.5 & 0.057 & $0.006 \%$ \\
\hline & 60 & $A 8$ & 15.8 & 0.057 & $0.006 \%$ \\
\hline & 250 & A9 & 7.5 & 0.057 & $0.006 \%$ o \\
\hline \multirow{3}{*}{$0.5 \mathrm{~ms}$ (disturbance) } & 15 & $B 1$ & 9.5 & 112 & $1.244 \%$ \\
\hline & 60 & $B 2$ & 8 & 84 & $0.933 \%$ \\
\hline & 250 & $B 3$ & 6.5 & 67 & $0.744 \%$ \\
\hline
\end{tabular}

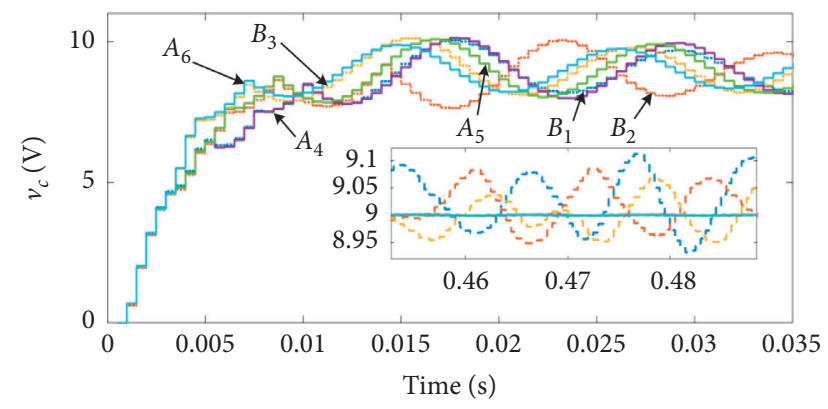

(a)

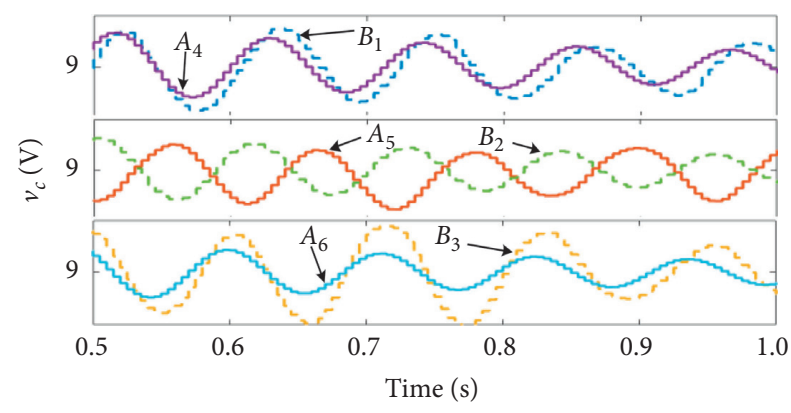

(b)

FIGURE 5: Output voltages affected by disturbances: (a) output voltages with different pairs of $(h, \lambda)$; (b) comparisons of local dynamics.

response times are almost the same and not affected by the lumped disturbance $d_{3}(x, \xi)$, but the values of steady error and relative steady error of the output voltages $v_{c}$ increase correspondingly. Furthermore, from the comparisons of local dynamics in Figure 5(b), their steady error changes and the parallel shift function imposed by disturbances are reconfirmed.

4.2. Experiment Results. In order to validate this paper, Figure 6(a) describes the structure of the experiment platform based on DSpace for the buck converter. The Rapid Control Prototype (RCP) system is composed of two parts, i.e., MicroAutoBox is used for collecting the voltage and current of the buck converter and RapidPro outputs the PWM signal for the direct control of the buck converter. Besides, they are linked with interface of Low-Voltage Differential Signaling (LVDS). The software of Matlab/ Simulink is installed in the host PC for developing algorithm following the program compiled by Real-Time Work (RTW) shop and then downloaded in MicroAutoBox. The real-time calculation is in DSpace 1401. The experiment flow diagram of DSAPCE is simplified in Figure 6(b), which is suitable for the algorithm design and performance analysis.

In order to prove the proposed unified approach to be suitable for diverse disturbances, four working modes of buck converters are selected as examples: (1) time-varying noise $0.05 \sin (2 \pi / h)$; (2) $\Delta V_{\text {ref }}=0.2 \mathrm{~V}$; (3) load resistor jumps 


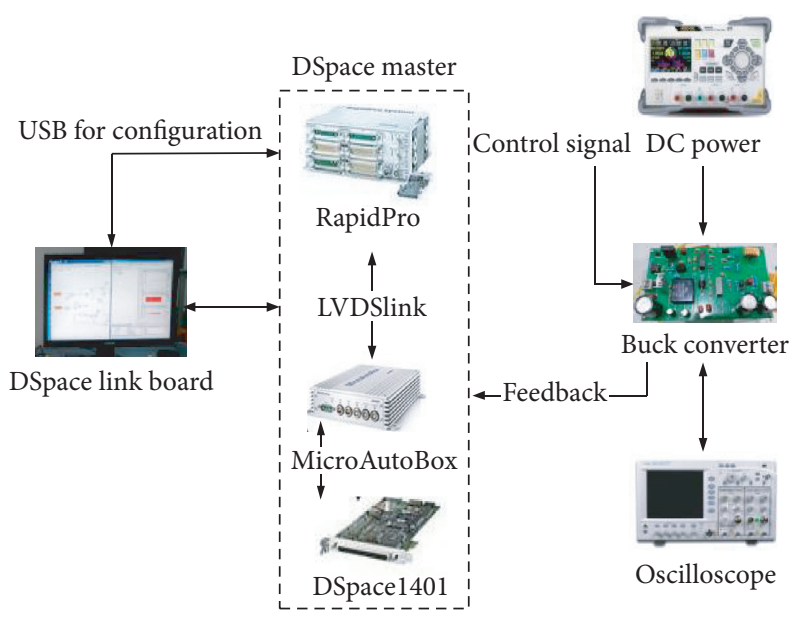

(a)

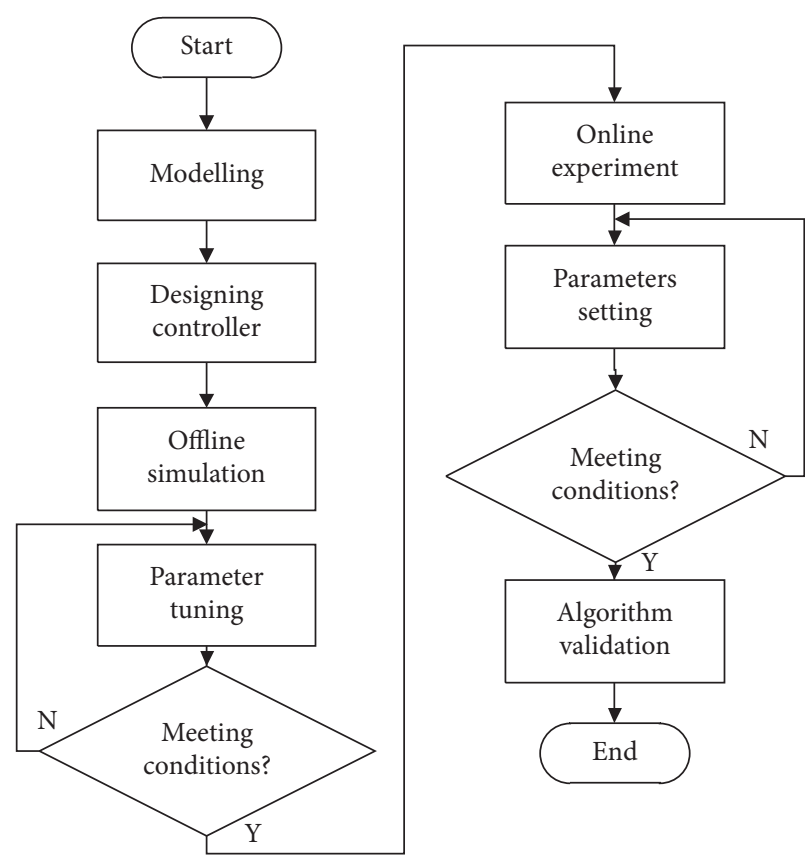

(b)

FIGURE 6: DSpace experiment platform of the buck converter system: (a) structure diagram of the experiment system; (b) experiment flow.

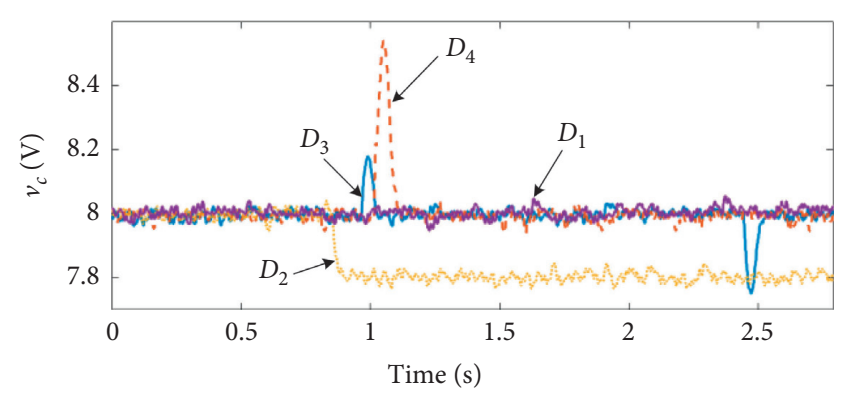

FIGURE 7: Experiment results of output voltages.

from $50 \Omega$ to $100 \Omega$ and then back to $50 \Omega$; and (4) input voltage changes from $16 \mathrm{~V}$ to $25 \mathrm{~V}$ and then back to $16 \mathrm{~V}$.

In experiment, the sampling time $h$ is chosen as $0.5 \mathrm{~ms}$. Referring to Table 2, the DTSM parameter $\lambda$ is chosen as 170 . And the contrastive experimental results are given in Figure 7 , where the output voltages of the four working modes are marked as $D 1, D 2, D 3$, and $D 4$, respectively. We can see that the DTSM controller can provide enough robustness to reject these disturbances. Despite the two peak values $8.18 \mathrm{~V}$ and $7.75 \mathrm{~V}$ for $D 3$ and one peak value $8.54 \mathrm{~V}$ for $D 4$, the steady errors of the four cases are listed as $0.32 \mathrm{~V}, 0.21 \mathrm{~V}$, $0.24 \mathrm{~V}$, and $0.26 \mathrm{~V}$, respectively, which are acceptable in practice. Therefore, the experiment results validate the proposed unified approach with wider applications under heavy uncertainty.

\section{Conclusions}

In this paper, the robust stability problem of DTSM controlled buck converters is investigated and a unified approach of modelling and controller design is proposed for all the possible model uncertainties and external disturbances. In modelling, two unified expressions are proposed to describe the possible individual/several/all disturbances. In the design of DTSM controller, the disturbances are directly included in the process, instead of ignoring them in the traditional approach. Innovatively, four more accurate subranges arise for the controller regulation. Based on the robust stability analysis, the influences of the sampling time and disturbances on the control system have also been investigated. Simulations and experiments validate the effectiveness and wider applications of the proposed approach.

\section{Data Availability}

The data used to support the findings of this study are included within the article.

\section{Conflicts of Interest}

The authors declare that they have no conflicts of interest regarding the publication of this paper.

\section{Acknowledgments}

This work was supported by the National Natural Science Foundation of China under Grants 5130703 and 61673132, and the authors would like to thank the experimental support from the 716 Research Institute, China Shipbuilding Industry Corporation.

\section{References}

[1] S. C. Siew-Chong Tan, Y. M. Lai, and C. K. Tse, "General design issues of sliding-mode controllers in DC-DC 
converters," IEEE Transactions on Industrial Electronics, vol. 55, no. 3, pp. 1160-1174, 2008.

[2] K. D. Young, V. I. Utkin, and U. Ozguner, "A control engineer's guide to sliding mode control," IEEE Transactions on Control Systems Technology, vol. 7, no. 3, pp. 328-342, 1999.

[3] A. G. Saman, G. S. Hosein, E. Mehran, and B. R. Ahmad, "Topologies and control schemes of bidrectional DC-DC converters: an overview," IEEE Access, vol. 7, pp. 117997118019, 2019.

[4] C. O. Mathúna, N. Ningning Wang, S. Kulkarni, and S. Roy, "Review of integrated magnetics for power supply on chip (PwrSoc)," IEEE Transactions on Power Electronics, vol. 27, no. 11, pp. 4799-4816, 2012.

[5] C.-Y. Chan, "A nonlinear control for DC-DC power converters," IEEE Transactions on Power Electronics, vol. 22, no. 1, pp. 216-222, 2007.

[6] S. Yang, D. Xiang, A. Bryant, P. Mawby, L. Ran, and P. Tavner, "Condition monitoring for device reliability in power electronic converters: a review," IEEE Transactions on Power Electronics, vol. 25, no. 11, pp. 2734-2752, 2010.

[7] L. Wang and M. S.-N. Thi, "Stability enhancement of largescale integration of wind, solar, and marine-current power generation fed to an SG-based power system through an LCCHVDC link," IEEE Transactions on Sustainable Energy, vol. 5, no. 1, pp. 160-170, 2014.

[8] J. Leyva-Ramos, J. A. Martinez-Cruz, and M. Martinez, "Robust stability analysis for current-programmed regulators," IEEE Transactions on Industrial Electronics, vol. 49, no. 5, pp. 1138-1145, 2002.

[9] J. Wang, S. Li, J. Yang, B. Wu, and Q. Li, "Extended state observer-based sliding mode control for PWM-based DC-DC buck power converter systems with mismatched disturbances," IET Control Theory \& Applications, vol. 9, no. 4, pp. 579-586, 2015.

[10] A. Khaligh, P. Chapman, J. Juri, and D. Ali, "Realization of parasitics in the stability of DC-DC converters loaded by constant-power loads in discontinuous conduction mode," IEEE Transactions on Industrial Electronics, vol. 19, no. 5, pp. 31-35, 2007.

[11] Y. Shtessel, S. Biglari, and B. Haik, "Unity power factor control in three-phase AC/DC boost converter using sliding modes," IEEE Transactions on Industrial Electronics, vol. 55, no. 11 , pp. 3874-3882, 2008.

[12] A. Francesco, D. I. Filippo, and C. Tommaso, "Identification and robust control of DC/DC converter hammerstein model," IEEE Transactions on Power Electronics, vol. 23, no. 6, pp. 2990-3003, 2008.

[13] S. Sharmila, O. Milijana, B. Serhiy, and B. Dushan, "Robust stability analysis of a DC/DC buck converter under multiple parametric uncertainties," IEEE Transactions on Power Electronics, vol. 33, no. 6, pp. 5426-5441, 2018.

[14] Y. Zhu and J. Fei, "Disturbance observer based fuzzy sliding mode control of PV grid connected inverter," IEEE Access, vol. 6, pp. 579-586, 2018.

[15] Y. Yin, J. Liu, A. S. Juan et al., "Observer-based adaptive sliding mode control of NPC converters: an RBF neural network approach," IEEE Transactions on Power Electronics, vol. 34, no. 4, pp. 483-497, 2019.

[16] Z. Xing, X. Ruan, and H. Xie, "PWM control scheme for a buck/boost modular multilevel DC/DC converter with reduced submodule capacitance," IEEE Transactions on Power Electronics, vol. 33, no. 5, pp. 3911-3925, 2018.

[17] S.-C. Tan, Y. M. Lai, M. K. H. Cheung, and C. K. Tse, "On the practical design of a sliding mode voltage controlled buck converter," IEEE Transactions on Power Electronics, vol. 20, no. 2, pp. 425-437, 2005.

[18] V. Repecho, D. Biel, J. M. Olm, and E. Fossas, "Robust sliding mode control of a DC/DC Boost converter with switching frequency regulation," Journal of the Franklin Institute, vol. 355, no. 13, pp. 5367-5383, 2018.

[19] H. Komurcugil, "Non-singular terminal sliding-mode control of DC-DC buck converters," Control Engineering Practice, vol. 21, no. 3, pp. 321-332, 2013.

[20] M. R. Seyed, Y. Monazzahalsadat, and H. Hassan, "Design of second order sliding mode and sliding mode algorithms: a practical insight to DC-DC buck converter," IEEE/CAA Journal of Automatica Sinica, vol. 4, no. 3, pp. 3831-3841, 2017.

[21] J. Yang, B. Wu, S. Li, and X. Yu, "Design and qualitative robustness analysis of an DOBC approach for DC-DC buck converters with unmatched circuit parameter perturbations," IEEE Transactions on Circuits and Systems I: Regular Papers, vol. 63, no. 4, pp. 551-560, 2016.

[22] J. Wang, S. Li, J. Yang, B. Wu, and Q. Li, "Finite-time disturbance observer based non-singular terminal sliding-mode control for pulse width modulation based DC-DC buck converters with mismached load disturbances," IET Power Electron.vol. 9, no. 9, pp. 1995-2002, 2016.

[23] Z. Wang, S. Li, and Q. Li, "Discrete-time fast terminal sliding mode control design for DC-DC buck converters with mismatched disturbances," IEEE Transactions on Industrial Informatics, vol. 16, no. 2, pp. 1204-1213, 2020.

[24] C. Wang, H. Xia, Y. Wang, Y. Mai, and S. Ren, "Discretisation performance analysis of sliding mode controlled DC-DC buck converter via zero-order holder," IET Control Theory \& Applications, vol. 13, no. 16, pp. 2583-2594, 2019.

[25] Z. Galias and X. Yu, "Analysis of delayed sliding mode control systems under zero-order holder discretization," IEEE Transactions on Automatic Control, vol. 61, no. 9, pp. 27392744, 2016.

[26] V. I. Enric, M. P. Adria, and G. Roberto, "Direct digital design of a sliding mode-based control of a PWM synchronous buck converter," IET Power Electron.vol. 13, no. 10, pp. 1714-1720, 2017.

[27] Z. Galias and X. Yu, "Analysis of zero-order holder discretization of two-dimensional sliding-mode control systems," IEEE Transactions on Circuits and Systems II: Express Briefs, vol. 55, no. 12, pp. 1269-1273, 2008.

[28] S. Janardhanan and B. Bandyopadhyay, "On discretization of continuous-time terminal sliding mode," IEEE Transactions on Automatic Control, vol. 51, no. 9, pp. 1532-1536, 2006.

[29] A. K. Behera and B. Bandyopadhyay, "Steady-state behaviour of discretized terminal sliding mode," Automatica, vol. 54, pp. 176-181, 2015.

[30] O. Huber, V. Acary, and B. Brogliato, "Lyapunov stability and performance analysis of theimplicitdiscrete sliding mode control," IEEE IEEE Transactions on Automatic Control, vol. 61, no. 10, pp. 3016-3030, 2015.

[31] X. Yu, B. Wang, Z. Galias, and G. Chen, "Discretization effect on equivalent control-based multi-input sliding-mode control systems," IEEE Transactions on Automatic Control, vol. 53, no. 6, pp. 1563-1569, 2008.

[32] B. Brogliato, A. Polyakov, and D. Efimov, "The implicit discretization of the supertwisting sliding-mode control algorithm," IEEE Transactions on Automatic Control, vol. 65, no. 8, pp. 3707-3713, 2019. 
[33] T. D. Wooley, "The cubic case of the main conjecture in Vinogradov's mean value theorem," Advances in Mathematics, vol. 294, pp. 532-561, 2016.

[34] Y. Feng, X. Yu, and Z. Man, "Non-singular terminal sliding mode control of rigid manipulators," Automatica, vol. 38, no. 12, pp. 2159-2167, 2002.

[35] M. Unser, "Sampling-50 years after Shannon," Proceedings of the IEEE, vol. 88, no. 4, pp. 569-587, 2000. 